See discussions, stats, and author profiles for this publication at: https://www.researchgate.net/publication/341521343

\title{
On the Contributions of Operational Flexibility Offered by Smart Sustainable Residential Buildings
}

Conference Paper · October 2020

DOI: 10.1109/ISGT-Europe47291.2020.9248929

3 authors:

Iason Avramidis

Luxembourg Institute of Science and Technology (LIST)

11 PUBLICATIONS 21 CITATIONS

SEE PROFILE

Florin Capitanescu

Luxembourg Institute of Science and Technology (LIST)

90 PUBLICATIONS 2,370 CITATIONS

SEE PROFILE

Geert Deconinck

KU Leuven

475 PUBLICATIONS 5,184 CITATIONS

SEE PROFILE

Some of the authors of this publication are also working on these related projects:

Project OOF 2003-15 View project

Project P2P SmarTest View project 


\title{
On the Contributions of Operational Flexibility Offered by Smart Sustainable Residential Buildings
}

\author{
Iason I. Avramidis* ${ }^{*}$, Florin Capitanescu*, and Geert Deconinck ${ }^{\dagger}$ \\ ${ }^{*}$ Environmental Research and Innovation, Luxembourg Institute of Science and Technology, Belvaux, Luxembourg \\ ${ }^{\dagger}$ Department of Electrical Engineering, KU Leuven/EnergyVille, Leuven, Belgium \\ Emails: [iason.avramidis, florin.capitanescu]@list.lu, geert.deconinck@kuleuven.be
}

\begin{abstract}
Most distribution systems, which were designed years ago under different standards, are largely incapable of integrating (in an uncoordinated manner) high levels of residential distributed energy resources (DER), becoming burdened by operational voltage and thermal issues. Especially for low voltage (LV) distribution systems, proper controllability is difficult, since the system operator rarely owns devices of its own. Customer-provided operational flexibility is expected to play an important role in maintaining proper operating conditions. This work examines various schemes to integrate high DER shares into LV networks by enlisting residential flexibility provided from all available residential devices. Emphasis is placed on the support potential by smart sustainable buildings (SSBs). A versatile multi-period optimal power flow (MP-OPF) model is developed, allowing for various levels of controllability of the available devices, referred to as flexibility resources (FRs). The model is tested on an $\mathrm{LV}$ feeder, in a day-ahead deterministic MP setting. It is demonstrated that when the available flexibility of SSBs is fully utilized the system is acceptably operated at minimum costs, coupled with minimum renewable curtailment. It is shown that this can be achieved without handing over full controllability of the FRs to the system operator.

Index Terms-Active Distribution Systems, Multi-Period Optimal Power Flow, Power System Flexibility, Smart Sustainable Building
\end{abstract}

\section{NOMENCLATURE}

\section{A. Sets}

$\begin{array}{ll}\mathcal{E} & \text { Set of electric vehicles }(\mathrm{EVs}) \\ \mathcal{I} & \text { Set of nodes } \\ \mathcal{P} & \text { Set of photovoltaic }(\mathrm{PV}) \text { panels } \\ \mathcal{S} & \text { Set of shiftable loads }(\mathrm{SLs}) \\ \mathcal{T} & \text { Set of time periods }\end{array}$

\section{B. Parameters}

$\xi \quad$ Sensitivity factor (set to 0.01 )

$C T_{s}$ Cycle time of SL $s$, time periods

$M^{P V}$ Maximum PV curtailment, \%

$M^{F L}$ Maximum FL alteration, \%

$I_{i j}^{\max }$ Line $i-j$ current limit, p.u.

$P_{e, t}^{c} \quad$ Nominal active power of EV $e$, period $t$, p.u.

$P_{i, t}^{D} \quad$ Active load demand, node $i$, period $t$, p.u.

$P_{p, t}^{g e n}$ PV $p$ generated active power, period $t$, p.u.

$P_{e / s}^{r a t e}$ Rated power (EV $e$ or SL $s$ ), p.u.

$Q_{i, t}^{D} \quad$ Reactive load demand, node $i$, period $t$, p.u.

$S_{i j}^{\text {max }}$ Transformer $i-j$ apparent power limit, p.u.

$t_{s}^{O N}$ Time period $t$ when SL $s$ normally turns "ON"

The authors acknowledge the funding from Luxembourg National Research Fund (FNR) in the framework of gENESiS project (C18/SR/12676686)

\section{Variables}

$P_{i j, t} \quad$ Active power flow at branch $i-j$, period $t$, p.u.

$P_{t}^{I E} \quad$ Active power import/export, period $t$, p.u.

$P_{p, t}^{i n j} \quad$ Active power injected by PV $p$, period $t$, p.u.

$P_{e, t}^{c, U}$ Active power "undercharge" of $\mathrm{EV} e$, period $t$, p.u.

$P_{e, t}^{c, O}$ Active power "overcharge" of EV $e$, period $t$, p.u.

$P_{i, t}^{D, U}$ Active power "underdemand", node $i$, period $t$, p.u.

$P_{i, t}^{D, O}$ Active power "overdemand", node $i$, period $t$, p.u.

$Q_{i j, t}$ Reactive power flow at branch $i-j$, period $t$, p.u.

$Q_{t}^{I E} \quad$ Reactive power import/export, period $t$, p.u.

$V_{i, t} \quad$ Voltage magnitude, node $i$, period $t$, p.u.

$\delta_{s, t} \quad$ Binary variable for SL $s$ at period $t ; \delta_{s, t}=1$ if the SL is "ON", otherwise $\delta_{s, t}=0$.

$\theta_{i, t} \quad$ Voltage angle difference, nodes $i, j$, period $t, \operatorname{rad}$

$\sigma_{i, t}^{u / d} \quad \mathrm{Up} /$ down voltage violation, node $i$, period $t$, p.u.

$\sigma_{i j, t} \quad$ Branch $i-j$ thermal limit violation, period $t$, p.u.

\section{INTRODUCTION}

Until recently, the design of low voltage (LV) distribution networks was based on the assumption that they would remain passive, hosting typical customer loads and a few local generators [1]. In the last few years, there has been a massive integration of distributed energy resources (DER), transforming distribution networks into increasingly active systems [2]. More often than not, they are inadequately designed for the uncoordinated deployment of such devices; distribution systems utilizing outdated infrastructure appear as weak systems. Most devices are customer-owned, and distribution system operators (DSOs) rarely have sufficient control over feeders, leading to many operational issues [3].

Operational issues can be mitigated by employing "static" approaches (grid reinforcement) or "active" approaches (demand response and flexibility utilization) [4]. However, the former are quite expensive and slow to deploy, while the latter require "heavy" customer participation or flexibility sources" ownership rights by the DSO. These assumptions are not very realistic, owing to a number of technical and legal barriers.

Voltage issues refer to instances of overvoltages or undervoltages, which can arise in the high presence of electricity producing, e.g., PV, and consuming, e.g., EV, devices respectively. Production curtailment or power factor adjustment are a few common approaches for dealing with overvoltages [5]. Conversely, undervoltages are dealt with on a per-case basis, depending on their "source". The aggregation and management of EV fleets was proposed in [6]. Direct DSO interference on the profiles of several electrical and thermal 
loads could also be an acceptable solution under proper controlability [7]. Bidirectional voltage control through energy storage is also an effective and encompassing solution [8].

An excellent resource of flexibility is the smart nearly zero energy (nZE) building, hereby referred to as smart sustainable building (SSB). An SSB balances on-site consumption and production throughout the year, and can provide extensive support to the distribution network [9]. The integration of SSBs in the power system is pushed by E.U. directives, with significant progress having been made in some countries, e.g., in Luxembourg [10]. On top of self-regulation, SSBs can also coordinate with the smart grid (under a vast array of goals) to provide broad support through a wide range of flexibility options. SSBs are concentrated pools of various forms of flexibility and can assist the DSO much better than individual devices scattered throughout the system.

This work introduces a novel, versatile multi-period optimal power flow (MP-OPF) framework, incorporating a vast array of residential flexibility resources (FRs, referring to devices that can provide flexibility to combat operational issues). Specific novel aspects of the proposed MP-OPF are the flexibility modelling and quantification with respect to nominal profiles and the reimbursement schemes for FLs, EVs and SLs. Unlike many previous works, the DSO is offered various levels of controllability over residential FRs. This work implicitly seeks to minimize deviations from the nominal FR profiles, rather than directly applying the optimal DSO-desired profiles, as is commonly the case. The MPOPF assesses the performance of all installed FRs that can be employed in modern smart LV distribution systems.

The remainder of this paper is structured as follows: Section II presents the assumptions and mathematical modelling. Section III presents the case study and the simulation results. Section IV offers conclusions and future work plans.

\section{Problem Formulation}

\section{A. Main assumptions}

The main assumptions of this work are highlighted below:

1) There is a bilateral agreement (flexible and adjustable [11]) between customers and a third-party entity (e.g., a specialized aggregator), allowing for partial alteration of the nominal FR customer-driven profiles, subject to appropriate remuneration. The entity, hereby referred to as "agent", explicitly represents the DSO's needs for operational issues management. Similar markets structures have been previously proposed (see [12]), though mainly for MV networks, where operational issues are fewer and the flexibility quantities procured are larger and easier to manage.

2) To explore the maximum benefit that FRs could offer, we assume that feeder's operation state can be estimated with sufficient accuracy. In addition, we assume the existence of communication links between controllable FRs and centralized computer that transmits new set-points to each The necessary measurement units and communication links are considered pre-installed.

3) The distribution feeder's behavior can be well approximated using the single-phase representation. Before transitioning to unbalanced settings, the proposed model must be first evaluated.
4) This work explores how effectively an existing flexibility setup can be utilized to manage operational issues. Said issues are assumed to be observed occasionally, not to a degree that would make grid reinforcement a financially viable option for shorter-term planning.

\section{B. Mathematical modelling}

1) Objective function: The DSO's objective is to maintain normal operating conditions for the optimization horizon (24-hour-ahead planning) at minimum cost (1). Based on assumption 2, real-time deviations are considered minimal or correctable with minimal cost, as better forecasting generally leads to fewer correction costs [13]. The approach is generic and applies for any optimization horizon and time resolution.

The total cost is comprised of three components: energy, capacity and violations (2)-(4). The slack component guarantees feasibility and quantifies the violations' severity. It is made up of a quadratic and a linear term, with the former demotivating higher violations, and the latter seeking to minimize "conflicts of interest" for the solver, when flexibility procurement and violations are equally acceptable. The various costs are represented by (5)-(9):

$$
\begin{gathered}
\text { minimize } \sum_{t \in \mathcal{T}}\left(E C_{t}+V C_{t}\right)+C C \\
E C_{t}=E C_{t}^{P V}+E C_{t}^{F L}+E C_{t}^{I E} \\
C C=C C^{E V}+C C^{S L} \\
V C_{t}=\left[\left(\sigma_{i, t}^{u}\right)^{2}+\left(\sigma_{i, t}^{d}\right)^{2}+\left(\sigma_{i j, t}\right)^{2}+\right. \\
\left.\sigma_{i, t}^{u}+\sigma_{i, t}^{d}+\sigma_{i j, t}\right] \cdot \phi \\
E C_{t}^{P V}=\sum_{p \in \mathcal{P}}\left(P_{p, t}^{g e n}-P_{p, t}^{i n j}\right) \cdot \alpha \\
E C_{t}^{F L}=\sum_{i \in \mathcal{I}}\left(P_{i, t}^{D, U} \cdot \epsilon+P_{i, t}^{D, O} \cdot \gamma\right) \\
C C^{E V}=\sum_{e \in \mathcal{E}, t \in \mathcal{T}}\left(\frac{P_{e, t}^{c, O}}{P_{e, t}^{c, U}+\xi}\right) \cdot P_{e}^{r a t e} \cdot \zeta \\
C C^{S L}=\sum_{s \in \mathcal{S}} P_{s}^{r a t e}\left(1-P_{s, t_{s}^{O N}}^{I E} \cdot \delta_{s,\left(t_{s}^{O N}+C T_{s}\right)}\right) \cdot \eta
\end{gathered}
$$

The energy costs comprise the PV curtailment cost (5), the cost of FL profile alteration (6) and the import/export cost (7).

EVs and SLs normally follow customer-defined, i.e., nominal profiles. Profile alterations, e.g., shifting of the SL to a different operating horizon, are reimbursed at a fixed, capacity-dependent commitment cost, regardless of the FR's "level of usage", see Eqs. (8)-(9). For SLs, the modelling of said setup is done using the already introduced SL binary variables, while for EVs, the barrier-function approach is employed. The selection of the barrier parameter $\xi$ must done with care, so as to represent the desired behavior without numerical issues, as observed in [14].

The flexibility utilization price per FR (Table I) is chosen based on a pre-decided desired activation priority order (PO). The PO depends on the setup and could be constructed by following a combination market rules and DSO/customer-driven factors, such as (among others) ease of flexibility activation, 
TABLE I: Characteristics of available FRs

\begin{tabular}{|c|c|c|c|}
\hline Service & PO & Symbol & Cost $(€ / \mathrm{kW})$ \\
\hline PV production curtailment & 6 & $\alpha$ & 25 \\
\hline Energy import/export & 1 & $\beta$ & 3 \\
\hline FL consumption increase & 3 & $\gamma$ & 7 \\
\hline FL consumption decrease & 2 & $\epsilon$ & 6 \\
\hline EV commitment cost & 5 & $\zeta$ & 15 \\
\hline SL commitment cost & 4 & $\eta$ & 10 \\
\hline Technical violations & 7 & $\phi$ & $50(€ / \mathrm{p} . \mathrm{u})$ \\
\hline
\end{tabular}

$$
\begin{array}{r}
P_{i j, t}=-V_{i, t}^{2} g_{i j}+V_{i, t} V_{j, t}\left[g_{i j} \cos \theta_{i j, t}+b_{i j} \sin \theta_{i j, t}\right] \\
Q_{i j, t}=V_{i, t}^{2} b_{i j}+V_{i, t} V_{j, t}\left[g_{i j} \sin \theta_{i j, t}-b_{i j} \cos \theta_{i j, t}\right] \\
\sum P_{i, p, t}^{i n j}+\sum P_{t}^{I E}-\sum\left[P_{i, t}^{D}+P_{i, t}^{D, O}-P_{i, t}^{D, U}\right] \\
-\sum P_{i, s}^{r a t e} \cdot \delta_{s, t}-\sum\left[P_{e, t}^{c}+P_{e, t}^{c, O}-P_{e, t}^{c, U}\right] \\
=\sum P_{i j, t} \begin{array}{c}
\sum \\
\sum Q_{t}^{I E}-0.3 \cdot \sum\left[P_{i, t}^{D}+P_{i, t}^{D, O}-P_{i, t}^{D, U}\right]=\sum Q_{i j, t} \\
V_{i, t}^{\min }-\sigma_{i, t}^{d} \leq V_{i, t} \leq V_{i, t}^{\max }+\sigma_{i, t}^{u} \\
P_{i j, t}^{2}+Q_{i j, t}^{2} \leq\left(S_{i j, t}^{\max }\right)^{2}+\sigma_{i j, t} \\
P_{i j, t}^{2}+Q_{i j, t}^{2} \leq V_{i, t}^{2}\left(I_{i j, t}^{\max }\right)^{2}+\sigma_{i j, t}
\end{array}
\end{array}
$$

Eqs. (17)-(18) are the branch power flow equations. Eqs. (19)-(20) are the nodal power balance constraints. Eqs. (21)(23) describe the (soft) technical limitations on voltage magnitudes, transformer power and line current limits respectively. The slack variables are non-negative.

\section{CASE STUdY}

mandatory and continuous cycle time Eqs. (11)-(12), meaning they have a fixed pattern that can be sifted through time. Examples include washing machines or water heaters. There are also time-frames during which the SL may not operate (here early morning and late night). A unity power factor (pf) is assumed. The modelling was originally proposed in [14]:

$$
\begin{array}{r}
\sum_{t \in \mathcal{T}} \delta_{s, t}=C T_{s} \quad \forall s \in \mathcal{S} \\
\sum_{t \in \mathcal{T}}\left[\delta_{s, t+1} \cdot\left(\delta_{s, t+1}-\delta_{s, t}\right)\right] \leq 1 \quad \forall s \in \mathcal{S}
\end{array}
$$

FLs (1 kW nominal power) can slightly alter their consumptions based on the DSO's needs, (13), without the need for energy shifting, as in the SL case. In other words, there is no strict pattern to be followed. Examples include AC units or heat pumps. These slight alterations should be compliant with the residents' comfort level [15]. Parameter $M^{F}$ is assumed to take that mandate into account. A pf of 0.95 is assumed:

$$
0 \leq P_{i, t}^{D, U}, P_{i, t}^{D, O} \leq P_{i, t}^{D} \cdot M^{F} \quad \forall i \in \mathcal{I}, \forall t \in \mathcal{T}
$$

EVs ( $3 \mathrm{~kW}$ rated power, $24 \mathrm{kWh}$ capacity) have customerdefined charging profiles. With respect to that, an EV can "overcharge" or "undercharge", based on the DSO's needs. An EV must be fully charged by the start of the new day, and the charging profile must not violate any technical limitations, (14)-(16). EVs may only charge when the resident is at home (here before 7 a.m. and after 6 p.m.). A unity pf is assumed:

$$
\begin{aligned}
\sum_{t \in \mathcal{T}}\left(P_{e, t}^{c, O}-P_{e, t}^{c, U}\right) & =0 \quad e \in \mathcal{E} \\
P_{e, t}^{c}+P_{e, t}^{c, O} \leq P_{e}^{r a t e} & \forall e \in \mathcal{E}, \forall t \in \mathcal{T} \\
P_{e, t}^{c}-P_{e, t}^{c, U} \geq 0 & \forall e \in \mathcal{E}, \forall t \in \mathcal{T}
\end{aligned}
$$

Lastly, the power system operational constraints must also hold $\forall t \in \mathcal{T}, \forall i, j \in \mathcal{I}: i \neq j, \forall e \in \mathcal{E}, \forall p \in \mathcal{P}, \forall s \in \mathcal{S}$ :

\section{A. Test system and "normal" profiles}

The proposed approach is applied on the benchmark Cigre 18-node LV feeder [16], as presented in Fig. 1. Nodes 11, 16, 18 are equipped with FLs and an EV, PV and SL respectively. SSBs are connected to nodes 15,17 . Note the in this context, we consider only electrical devices, while the "sustainability" of SSBs is expressed in terms of their yearly nZE target.

Typical profiles of the various FRs for a typical autumn day were utilized, adopted from [17]. The SSB profiles are assumed to have been calculated internally by EMS systems and are derivative of their yearly planned profiles, which adhere to the nZE mandate. As such, the daily SSB profiles do not explicitly need to adhere to said mandate. The aim of the analysis is not to perform a season-dependent system stress test, but to rather explore the flexibility potential of various FRs in systems with high DER penetration. Different flexibility scenarios are examined (see Table I), where the agent's range of "controlability" progressively expands.

The solved problem can be either MINLP or NLP, depending in whether or not SL are considered as flexibility options. Both cases are modelled in GAMS [18] and solved by BONMIN [19] and IPOPT [20], respectively.

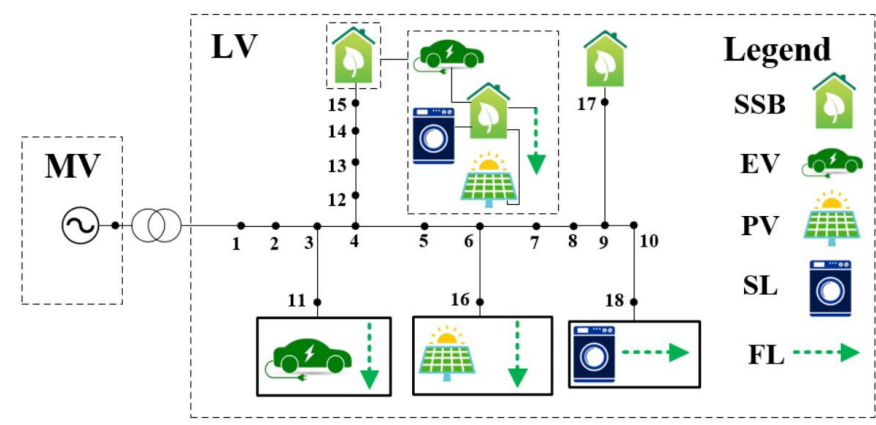

Fig. 1: Layout of 18-node LV feeder 


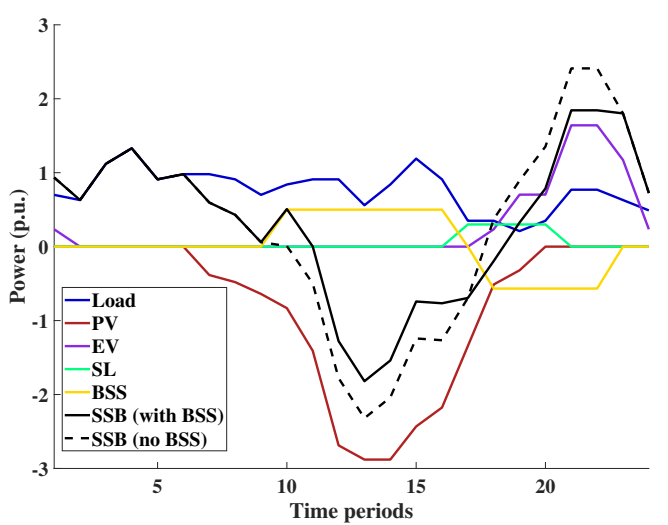

Fig. 2: Sum of power profiles for each FR (1 kW basis)

TABLE II: Examined flexibility scenarios

\begin{tabular}{|c|c|c|c|}
\hline FR & Cost & $M^{P V}$ & $M^{F L}$ \\
\hline S1 & - & 0 & 0 \\
\hline S2/S3/S4 & PV & $0.25 / 0.5 / 0.75$ & 0 \\
\hline S5/S6/S7 & PV, FL & 0.75 & $0.1 / 0.2 / 0.3$ \\
\hline S8 & PV, FL, SL & 0.75 & 0.3 \\
\hline S9 & PV, FL, SL, EV & 0.75 & 0.3 \\
\hline
\end{tabular}

\section{B. Simulation Results}

The profiles of the maximum and minimum voltages in the network are presented in Figs. 3 and 4 respectively. High violations are initially observed, with overvoltages of over 0.25 p.u. and undervoltages of over 0.2 p.u.. Enlisting the aid of residential flexibility appears mandatory, as the system cannot be properly operated when any single FR is unavailable, thus preventing large DER deployment.

As the integration of flexibility increases, the severity of voltage issues decreases, and the total impact on the voltage profile across the network is contained. In fact, as available PV curtailment starts reaching the $75 \%$ mark, represented by $\mathrm{S} 4$ in Fig. 5, overvoltages are gradually eliminated. The subsequent availability of FLs (S5-S7) assists in reducing undervoltages, after which the shifting of the SLs (S8) and the "rescheduling" of the EVs' (S9) charging profiles also eliminate all undervoltages. As compared to the noflexibility scenario, total improvements of approximately 3.8 p.u. and 3.2 p.u. are achieved across the network in combating overvoltages and undervoltages respectively (see Fig. 5).

The flexibility utilisation costs are presented in Fig. 6.

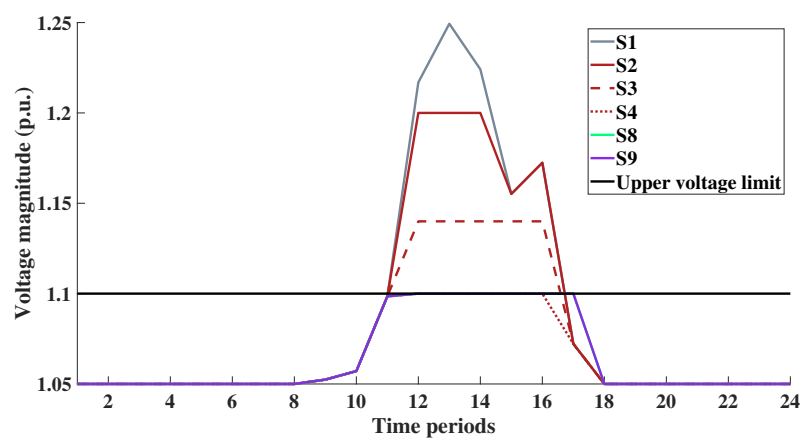

Fig. 3: Maximum voltage per time period
Initially, overvoltages can be countered by exporting excess energy (this quickly "saturates") or by curtailing PV, with curtailment and undervoltage violation costs being very high. As FL becomes available, containing overvoltages is cheaper, and PV curtailment is avoided. The FL also helps in mitigating undervoltages at different periods. As SLs become available, overvoltages and undervoltages are combated simultaneously, since the SLs' operation is shifted to coincide with periods of high PV production, which also alleviates some of the stress from previously consumption-heavy periods. Lastly, as EVs become available, the final consumption-heavy periods are spread throughout the day (note that EVs are not connected to the customer's charging station during periods of high PV production), thus mitigating the last undervoltage issues.

For the most "inclusive" flexibility scenario, all FRs are employed, eliminating all initially observed voltage issues (no violation costs). However, the PV curtailment costs continue to take up a significant percentage of the total costs (more than 60\%); this makes the case that the inclusion of BSS in systems with high renewable penetration could be beneficial, even if the agent does not have full operational control. This is because the customers' own decisions over such versatile devices could be inherently beneficial for the system.

It is worth highlighting the contribution of SSBs. As can be glimpsed from Fig. 6 (excluding slack and IE costs), the flexibility costs attributed to SSB participation makes up roughly $60 \%-70 \%$ of the total costs. This is in accordance with the SSBs' "presence" in the system: SSBs are responsible for approximately $65 \%$ of the available flexibility stemming from PVs, EVs and SLs, and for approximately $40 \%$ of the available flexibility stemming from FLs. However, contrary to other customers, SSBs always support the system in some

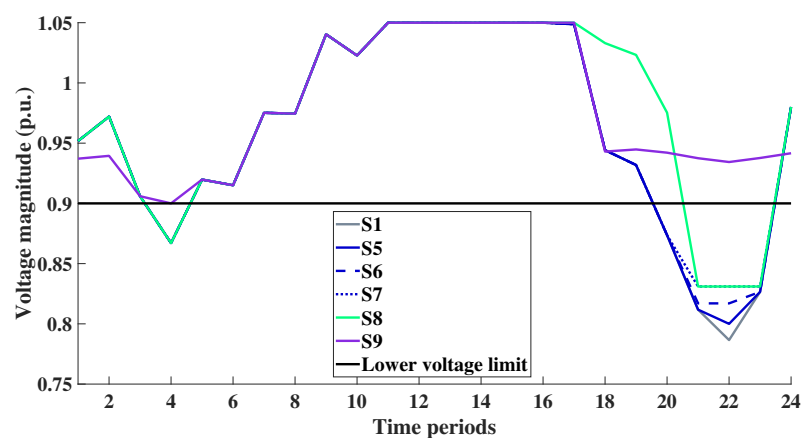

Fig. 4: Minimum voltage per time period

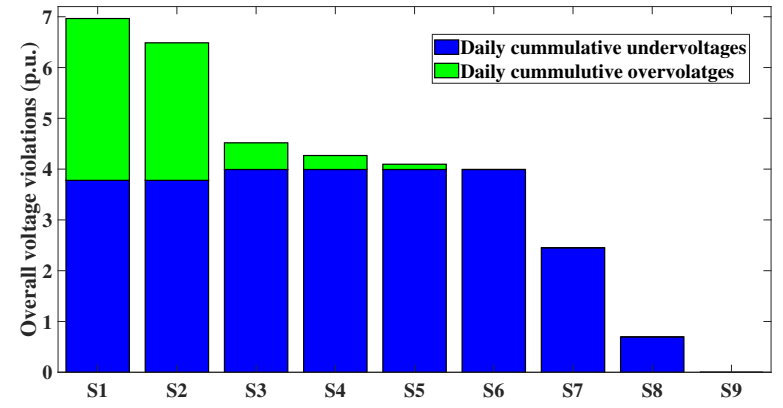

Fig. 5: Cumulative voltage improvement per scenario 




Fig. 6: Flexibility costs per scenario: Left bars are the total costs, right bars are the SSB costs

fashion, while directly causing (on average) fewer issues. This makes them "powerful" assets at the agent's disposal.

While slightly outside the paper's scope, we present a preliminary demonstration of how the mere existence of BSS could significantly ameliorate the situation. In Fig. 7, we compare the costs between two different scenarios: the most "inclusive" flexibility scenario (as presented in Fig. 4) and the same scenario but with the addition of residential battery storage systems (BSS) in the SSBs. The BSS $(2 \mathrm{~kW}$ rated power, $6.4 \mathrm{kWh}$ capacity) follow an intuitive operational pattern, charging at 0.5 p.u. between 8 a.m. and 4 p.m. (expected PV production) and discharging at 0.567 p.u. between 6 p.m. and 10 p.m.. This is manually set by the customer and the agent has no controllability over the BSS profile.

The mere existence of BSS drastically improves system conditions, also decreasing procurement costs by over $60 \%$. Even a rule-based approach for operating BSS offers bidirectional solutions for mitigating voltage issues. EVs are largely serviced by the discharging of the BSS, thus combating many of the original undervoltages. PVs have effectively been coupled with a "sink" for their excess production and almost all of the original PV curtailment has now been avoided. The connection of BSS in the feeder reduces the agent's need to resort to customer-provided flexibility. Do also note that this is largely due to the existence of SSBs, which are ideal candidates for hosting BSS, as individual ownership is currently extremely limited. While full BSS controllability could further improve the results, it is a scenario that is extremely unlikely to occur.



Fig. 7: Flexibility cost comparison: with BSS vs without BSS

\section{CONCLusions \& Future Work}

This paper presents a comprehensive MP-OPF model, which incorporates several novel modeling aspects, and various residential FRs which can easily be included into any flexibility scheme. It is shown that when the distribution system is heavily loaded with uncontrollable (potential) FRs, voltage issues are almost certain to naturally occur, thus highlighting the necessity of residential flexibility. The importance of SSBs is also demonstrated, being excellent sources of flexibility due to operating a vast array of flexible options.

Apart from the logical extension to unbalanced networks, the authors plan to propose a comprehensive framework the properly defines the FR remuneration scheme and PO rather than relying on simplistic and empirical assumptions.

\section{REFERENCES}

[1] J.A. Peças Lopes et al. 'Integrating Distributed Generation into Electric Power Systems: A Review of Drivers, Challenges and Opportunities". Electric Power Systems Research, 77(9):1189-1203, 2007.

[2] A. Keane et al. "State-of-the-Art Techniques and Challenges Ahead for Distributed Generation Planning and Optimization". IEEE Trans. Power Syst., 28(2):1493-1502, 2013.

[3] I.I. Avramidis et al. "Demand Side Flexibility Schemes for Facilitating the High Penetration of Residential Distributed Energy Resources". IET Gener. Transm. Distr., 12(18):4079-4088, 2018.

[4] S. Hashemi and J. Østergaard. "Methods and Strategies for Overvoltage Prevention in Low Voltage Distribution Systems with PV". IET RPG, 11(2):205-214, 2017.

[5] J. Leon, A. Padilha-Feltrin, J. Melo, and A. Filho. "Voltage control in low voltage distribution networks with high penetration photovoltaic system". In ISGT Latin America, September 20-22, 2017.

[6] S. Vandael et al. "A Scalable Three-Step Approach for Demand Side Management of Plug-in Hybrid Vehicles". IEEE Trans. Smart Grid, 4(2):720-728, 2013.

[7] I.D. de Cerio Mendaza, I.G. Szczesny, J.R. Pillai, and B. Bak-Jensen. "Flexible Demand Control to Enhance the Dynamic Operation of Low Voltage Networks". IEEE Trans. Smart Grid, 6(2):705-714, 2015.

[8] Y. Wang et al. "Coordinated Control of Distributed Energy-Storage Systems for Voltage Regulation in Distribution Networks". IEEE Trans. Power Del., 31(3):1132-1141, 2016.

[9] A.J. Marszal et al. "Zero Energy Building-A Review of Definitions and Calculation Methodologies". Energy and Buildings, 43(4):971979, 2011.

[10] S. Rafii-Tabrizi, J.R, Hadji-Minaoglou, F. Scholzen, F. Capitanescu. "Optimal Operation of Nearly Zero Energy Buildings using Mixed Integer Linear Programming”. In SEST Portugal, 2019.

[11] C. Kok et al. "A DSO-Level Contract Market for Conditional Demand Response". In IEEE Milan Powertech, June 23-27, 2019.

[12] C. Zhang et al. "FLECH: A Danish market solution for DSO congestion management through DER flexibility services". Journal of Modern Power Systems and Energy, 2(2):126-133, 2014.

[13] V.A. Evangelopoulos et al. "Flexibility Services Management under Uncertainties for Power Distribution Systems: Stochastic Scheduling and Predictive Real-time Dispatch". IEEE Access, 8, 2020.

[14] I.I. Avramidis, F. Capitanescu, and G. Deconinck. "A Tractable Approximation Approach for Dealing with the Binary Nature of Shiftable Loads in Multi-Period Optimal Power Flow". In IEEE Texas Power and Energy Conference, February 6-7, 2020.

[15] A. Anvari-Moghaddam, H. Monsef, and A. Rahimi-Kian. "Optimal Smart Home Energy Management Considering Energy Saving and a Comfortable Lifestyle". IEEE Trans. Smart Grid, 6(1):324-332, 2014.

[16] K. Strunz et al. "Benchmark Systems for Network Integration of Renewable and Distributed Energy Resources". CIGRE, Task Force C6.04, pages 4-6, 2014.

[17] A. Navarro-Espinosa and L.F. Ochoa. "Probabilistic impact assessment of low carbon technologies in LV distribution systems". IEEE Trans. Power Syst., 31(3):2192-2203, 2015.

[18] B.A. McCarl, GAMS user guide, version 23.8, 2012, www.gams.com.

[19] P. Bonami et al. "An Algorithmic Framework for Convex Mixed Integer Nonlinear Programs". Discrete Optimization, 5(2):186-204, 2008.

[20] A. Wächter and L. T. Biegler. "On the Implementation of a Primal-Dual Interior Point Filter Line Search Algorithm for Large-Scale Nonlinear Programming". Mathematical Programming, 106(1):25-57, March 2006. 